\title{
Retrodiction with two-level atoms: atomic previvals
}

\author{
John Jeffers ${ }^{1}$, Stephen M. Barnett ${ }^{1}$ and David T. Pegg ${ }^{2}$ \\ ${ }^{1}$ Department of Physics and Applied Physics, University of Strathclyde, Glasgow G4 ONG, \\ United Kingdom. \\ ${ }^{2}$ Faculty of Science, Griffith University, Nathan, Brisbane, Q111, Australia.
}

(February 1, 2008)

\begin{abstract}
In the Jaynes-Cummings model a two-level atom interacts with a single-mode electromagnetic field. Quantum mechanics predicts collapses and revivals in the probability that a measurement will show the atom to be excited at various times after the initial preparation of the atom and field. In retrodictive quantum mechanics we seek the probability that the atom was prepared in a particular state given the initial state of the field and the outcome of a later measurement on the atom. Although this is not simply the time reverse of the usual predictive problem, we demonstrate in this paper that retrodictive collapses and revivals also exist. We highlight the differences between predictive and retrodictive evolutions and describe an interesting situation where the prepared state is essentially unretrodictable.
\end{abstract}

PACS number(s): 42.50.Md, 03.65.Wj, 03.65.Ca

Typeset using REVTEX 


\section{INTRODUCTION}

The rapid development of quantum information theory in recent years has given fresh impetus to the study of retrodictive quantum theory. A retrodictive quantum formalism was first proposed more than 30 years ago, and others have followed [四]. Recently its utility has been extended by the application of Bayes' theorem [2] to the conditional probabilities derived using predictive quantum theory [3]. The theory applies to closed systems, and also to open systems in which the system of interest interacts with an unmeasured environment [1] 6 .

Normally we want to predict the future based on our knowledge of the present, so in predictive quantum theory the state of the system at any time between preparation and measurement is the evolved prepared state. Sometimes, however, our knowledge of initial states is not complete. If we know the result of a measurement of the state we can assign a retrodictive state on the basis of the measurement outcome. Each measurement outcome has associated with it a probability operator measure (POM) element [7]. It is possible to prove using Bayes' theorem [2] that the retrodictive density operator is simply the normalised POM element [3]. For closed systems this state evolves backwards in time to the preparation time according to the Schrödinger equation, when it collapses on to one of a set of possible initially prepared states. Normally the predictive and retrodictive states assigned to a system at a particular time between preparation and measurement will be different.

For open systems the evolution is more complicated. The simple time-reversal property of closed systems does not apply. In general the system to be measured interacts with another unmeasured system usually called the environment, which is traced out of the problem to give the (nonunitary) evolution of the system of interest. This evolution is governed by a master equation for the system density operator. We have recently derived methods for solving retrodictive problems in open systems based on standard predictive master equation techniques [4. Furthermore, we have derived a retrodictive master equation which traces the evolution of such systems backwards in time [6]. 
These master equation methods have been used to prove that optical amplifiers and attenuators are predictive/retrodictive inverses of one another [5]. Also, retrodiction from measured atomic states, for a two-level atom interacting with an environment which consists of a multimode electromagnetic field, has been studied [4]. This system illustrates various general principles of retrodictive open systems. In particular, if nothing is known about the initial state of the system the retrodictive steady state is usually the no-information state. This is an equally-weighted mixture of all the possible input states. In addition, the retrodictive decay rate depends upon the measurement outcome. There are also properties peculiar to this two-level system. If the atom is illuminated with coherent light retrodictive Rabi oscillations occur, which decay eventually to the no-information state.

In this paper we concentrate in particular on the two-level atom interacting with a single cavity mode of the quantised electromagnetic field. In standard predictive quantum theory, if the evolution of the atomic state is driven by a known coherent field, Rabi oscillations occur both in the atomic population, and in the off-diagonal atomic coherences. The frequency of the oscillations increases with the square root of the number of quanta of energy in the system. For a coherent driving field the number of photons is not completely certain; rather the field is in a weighted superposition of all photon number states. After a short time the different Rabi oscillations for each of the number states within the superposition get out of phase with one another and the oscillations of the whole system collapse. Later, when sufficient time has elapsed so that the oscillations get back into phase, there is a revival of the oscillating atomic population [8 10]. Here we look at this system from the retrodictive point of view, where we measure the final state of the atom knowing nothing about its initial state. The field is initially in a coherent state, but it is not measured after the interaction. The retrodictive situation is not the time-reverse of the predictive situation because we have different knowledge in the two cases. In the retrodictive situation we know the initial state of the field and the final measured state of the atom, whereas in the predictive case these states are both known at the initial time. This poses an interesting question. Would we retrodict that the retrodictive Rabi oscillations were in phase at times prior to the measurement of 
the field state, that is, would we retrodict any "previvals"?

The paper is organised as follows. In Section (II) we briefly describe the general features of retrodictive quantum theory, and its application to the two-level atom interacting with an electromagnetic field. We apply this in Section (III) and give results for the retrodictive density matrix and preparation probabilities. Section (IV) contains a summary and discussion of the main results of the paper.

\section{PREDICTION AND RETRODICTION}

In this section we provide brief details of retrodictive quantum theory. A fuller account can be found in references [3] and [4]. Suppose that we have a preparation device which

produces output states $\hat{\rho}_{i}^{\text {pred }}$ with prior probabilities $P(i)$, where $\hat{\rho}_{i}^{\text {pred }}$ is the usual density operator of predictive quantum mechanics. This state can evolve and interact with other systems until it is measured by a measuring device. A general description of a measurement is given by a measurement POM [7]. This is a set of non-negative definite, Hermitian elements $\hat{\Pi}_{j}$ which sum to the unit operator, each element corresponding to a particular measurement outcome. In general there is no requirement that there be the same number of POM elements as there are states which span the system space, but for von Neumann measurement this is so, and the POM elements are simply the projectors of the particular chosen states which span the space. Suppose that preparation takes place at time $t_{\mathrm{p}}$ and measurement at a later time $t_{\mathrm{m}}$. Within this framework the predictive probability that the measurement outcome $\hat{\Pi}_{j}$ is obtained given that the state $\hat{\rho}_{i}^{\text {pred }}$ was prepared is

$$
P(j \mid i)=\operatorname{Tr}\left(\hat{\rho}_{i}^{\operatorname{pred}}\left(t_{\mathrm{m}}\right) \hat{\Pi}_{j}\right)
$$

where

$$
\hat{\rho}_{i}^{\operatorname{pred}}\left(t_{\mathrm{m}}\right)=\hat{U}(\tau) \hat{\rho}_{i}^{\text {pred }}\left(t_{\mathrm{p}}\right) \hat{U}^{\dagger}(\tau)
$$

is the evolved initial density operator and 


$$
\hat{U}(\tau)=\exp \left(-\frac{i}{\hbar} \hat{H} \tau\right)
$$

is the evolution operator, which operates for the length of time between preparation and measurement, $\tau=t_{\mathrm{m}}-t_{\mathrm{p}}$.

Suppose that instead of calculating the predictive probability $P(j \mid i)$ we wish to calculate the retrodictive conditional probability $P(i \mid j)$ that the state $\hat{\rho}_{i}^{\text {pred }}$ was prepared, given our measurement result $\hat{\Pi}_{j}$. It is possible to do this by calculating all possible predictive conditional probabilities for the system, and then using Bayes' theorem. A simpler and more natural approach is to use retrodictive quantum theory, so that the required probability can be written [3,4]

$$
P(i \mid j)=\frac{\operatorname{Tr}\left[\hat{\Lambda}_{i} \hat{\rho}_{j}^{\text {retr }}\left(t_{\mathrm{p}}\right)\right]}{\operatorname{Tr}\left[\hat{\Lambda} \hat{\rho}_{j}^{\text {retr }}\left(t_{\mathrm{p}}\right)\right]} .
$$

Here the operator $\hat{\Lambda}_{i}$ is the preparation device operator, and

$$
\hat{\Lambda}=\sum_{i} \hat{\Lambda}_{i}=\sum_{i} P(i) \hat{\rho}_{i}^{\text {pred }}
$$

is the a priori density operator, the sum of each possible preparation density operator weighted by its prior probability of production. $\hat{\Lambda}$ is the best description of the state we can give without knowing the outcome of the preparation or measurement. The retrodictive density operator at the preparation time is simply the normalised measurement POM element evolved back from the measurement time to the preparation time,

$$
\hat{\rho}_{j}^{\mathrm{retr}}\left(t_{\mathrm{p}}\right)=\hat{U}^{\dagger}(\tau) \hat{\rho}_{j}^{\mathrm{retr}}\left(t_{\mathrm{m}}\right) \hat{U}(\tau),
$$

with

$$
\hat{\rho}_{j}^{\text {retr }}\left(t_{\mathrm{m}}\right)=\frac{\hat{\Pi}_{j}}{\operatorname{Tr} \hat{\Pi}_{j}} .
$$

The above formulae for the conditional probabilities (eqs. (11) and (4)) apply equally well for open systems, where the system of interest interacts with an unmeasured environment with many degrees of freedom. If this environment causes information to be lost about the 
system, and the Born-Markov approximation holds, then the the evolved density operators are the solutions of master equations [11]. In eq.(11) the density operator required for such an open system, which for a closed system would be given by eq. (2), is the solution of the usual master equation forwards in time from the preparation time to the measurement time. However, in eq.(1) the solution required instead of eq. (6) is that of the retrodictive master equation, giving the evolution backwards in time from the measurement time to the preparation time. We have recently derived this equation from the general principle that conditional probabilities should be independent of the time of collapse of the wavefunction [6]. In the system considered in the present paper, however, the Born-Markov approximation is not made, and so we must consider the full evolution of the coupled atom-field system.

\section{RETRODICTION FOR THE COUPLED ATOM-FIELD SYSTEM}

Here we apply the retrodictive formalism to a coupled system consisting of a two-level atom and a single cavity mode of an electromagnetic field. The interaction between an atom with upper level $|e\rangle$ and lower level $|g\rangle$, and an electromagnetic field is governed by the Jaynes-Cummings Hamiltonian [11]. In the interaction picture this is

$$
\hat{H}=\frac{\hbar \Delta}{2} \hat{\sigma}_{3}-i \hbar \lambda\left(\hat{\sigma}_{+} \hat{a}-\hat{a}^{\dagger} \hat{\sigma}_{-}\right)
$$

where $\Delta$ is the detuning between the atomic frequency and the light, $\hat{\sigma}_{3}=|e\rangle\langle e|-| g\rangle\langle g|$ is the atomic inversion operator, $\hat{\sigma}_{+}=|e\rangle\langle g|$ and $\hat{\sigma}_{-}=|g\rangle\langle e|$ are the atomic raising and lowering operators, $\hat{a}^{\dagger}$ and $\hat{a}$ are the creation and annihilation operators for the single mode field, and $\lambda$ is the coupling constant. The rotating wave approximation, which has been made in deriving this Hamiltonian, ensures that whenever a photon is lost from the field the atomic state must change from $|g\rangle$ to $|e\rangle$. In the standard predictive picture of quantum mechanics a coupled atom-field system evolves forwards in time according to this Hamiltonian from a preparation time $t_{\mathrm{p}}$ to a measurement time $t_{\mathrm{m}}$. After this has happened the coupled density operator for the whole system is 


$$
\hat{\rho}_{\mathrm{af}}^{\mathrm{pred}}\left(t_{\mathrm{m}}\right)=\hat{U}(\tau) \hat{\rho}_{\mathrm{a}}^{\mathrm{pred}}\left(t_{\mathrm{p}}\right) \otimes \hat{\rho}_{\mathrm{f}}^{\mathrm{pred}}\left(t_{\mathrm{p}}\right) \hat{U}^{\dagger}(\tau)
$$

where $\hat{\rho}_{\text {af }}^{\text {pred }}\left(t_{\mathrm{m}}\right)$ is the coupled density operator for the atom-field system at the measurement time, $\hat{\rho}_{\mathrm{a}}^{\text {pred }}\left(t_{\mathrm{p}}\right)$ and $\hat{\rho}_{\mathrm{f}}^{\text {pred }}\left(t_{\mathrm{p}}\right)$ are the uncoupled atom and field density operators at the preparation time. $\hat{U}(\tau)$ is the evolution operator for the coupled system, given by eq.(3) with Hamiltonian given by eq. (8). If the field is unmeasured the atomic state is simply found by tracing over field states and vice versa,

$$
\begin{aligned}
& \hat{\rho}_{\mathrm{a}}^{\operatorname{pred}\left(t_{\mathrm{m}}\right)}=\operatorname{Tr}_{\mathrm{f}}\left[\hat{U}(\tau) \hat{\rho}_{\mathrm{a}}^{\operatorname{pred}}\left(t_{\mathrm{p}}\right) \otimes \hat{\rho}_{\mathrm{f}}^{\operatorname{pred}}\left(t_{\mathrm{p}}\right) \hat{U}^{\dagger}(\tau)\right], \\
& \hat{\rho}_{\mathrm{f}}^{\operatorname{pred}\left(t_{\mathrm{m}}\right)}=\operatorname{Tr}_{\mathrm{a}}\left[\hat{U}(\tau) \hat{\rho}_{\mathrm{a}}^{\operatorname{pred}}\left(t_{\mathrm{p}}\right) \otimes \hat{\rho}_{\mathrm{f}}^{\operatorname{pred}}\left(t_{\mathrm{p}}\right) \hat{U}^{\dagger}(\tau)\right] .
\end{aligned}
$$

Alternatively, we can condition the field by measuring the atom, or the atom by measuring the field. This is done using the POM element corresponding to the measurement outcome. Thus the conditioned atomic and field states immediately after the measurement are

$$
\begin{aligned}
& \hat{\rho}_{\mathrm{a}}^{\operatorname{pred}}\left(t_{\mathrm{m}}\right) \propto \operatorname{Tr}_{\mathrm{f}}\left[\hat{\Pi}_{\mathrm{f}} \hat{U}(\tau) \hat{\rho}_{\mathrm{a}}\left(t_{\mathrm{p}}\right) \otimes \hat{\rho}_{\mathrm{f}}\left(t_{\mathrm{p}}\right) \hat{U}^{\dagger}(\tau)\right], \\
& \hat{\rho}_{\mathrm{f}}^{\text {pred }}\left(t_{\mathrm{m}}\right) \propto \operatorname{Tr}_{\mathrm{a}}\left[\hat{\Pi}_{\mathrm{a}} \hat{U}(\tau) \hat{\rho}_{\mathrm{a}}\left(t_{\mathrm{p}}\right) \otimes \hat{\rho}_{\mathrm{f}}\left(t_{\mathrm{p}}\right) \hat{U}^{\dagger}(\tau)\right],
\end{aligned}
$$

where $\hat{\Pi}_{\mathrm{a}(\mathrm{f})}$ is the POM element corresponding to the outcome of the measurement performed on the atom (field).

The retrodictive picture differs from above in that the state of the system is assigned on the basis of the measurement outcome. Thus if the measurement POM elements for the atom and the field are $\hat{\Pi}_{\mathrm{a}}\left(t_{\mathrm{m}}\right)$ and $\hat{\Pi}_{\mathrm{f}}\left(t_{\mathrm{m}}\right)$, the coupled initial density operator corresponding to equation (9) is

$$
\begin{aligned}
\hat{\rho}_{\mathrm{af}}^{\mathrm{retr}}\left(t_{\mathrm{p}}\right) & =\hat{U}^{\dagger}(\tau) \hat{\rho}_{\mathrm{a}}^{\mathrm{retr}}\left(t_{\mathrm{m}}\right) \otimes \hat{\rho}_{\mathrm{f}}^{\mathrm{retr}}\left(t_{\mathrm{m}}\right) \hat{U}(\tau) \\
& \propto \hat{U}^{\dagger}(\tau) \hat{\Pi}_{\mathrm{a}}\left(t_{\mathrm{m}}\right) \otimes \hat{\Pi}_{\mathrm{f}}\left(t_{\mathrm{m}}\right) \hat{U}(\tau) .
\end{aligned}
$$

The atom or the field will in general have been prepared in one of a set of initial states, and this state conditions the coupled density operator similarly to equations (12) and (13), to give retrodictive density operators for the atom and field respectively 


$$
\begin{aligned}
& \hat{\rho}_{\mathrm{a}}^{\mathrm{retr}}\left(t_{\mathrm{p}}\right) \propto \operatorname{Tr}_{\mathrm{f}}\left[\hat{\rho}_{\mathrm{f}}^{\mathrm{pred}}\left(t_{\mathrm{p}}\right) \hat{U}^{\dagger}(\tau) \hat{\Pi}_{\mathrm{a}}\left(t_{\mathrm{m}}\right) \otimes \hat{\Pi}_{\mathrm{f}}\left(t_{\mathrm{m}}\right) \hat{U}(\tau)\right] \\
& \hat{\rho}_{\mathrm{f}}^{\mathrm{retr}}\left(t_{\mathrm{p}}\right) \propto \operatorname{Tr}_{\mathrm{a}}\left[\hat{\rho}_{\mathrm{a}}^{\mathrm{pred}}\left(t_{\mathrm{p}}\right) \hat{U}^{\dagger}(\tau) \hat{\Pi}_{\mathrm{a}}\left(t_{\mathrm{m}}\right) \otimes \hat{\Pi}_{\mathrm{f}}\left(t_{\mathrm{m}}\right) \hat{U}(\tau)\right]
\end{aligned}
$$

where the constant of proportionality is determined by normalisation. If there is no informa-

tion at all about the preparation of the initial states then $\hat{\rho}_{\mathrm{a}}^{\mathrm{pred}}\left(t_{\mathrm{p}}\right)$ and $\hat{\rho}_{\mathrm{f}}^{\mathrm{pred}}\left(t_{\mathrm{p}}\right)$ become proportional to the unit operators for the atom and the field.

\section{RETRODICTION OF THE ATOMIC STATE: COLLAPSES AND PREVIVALS}

Here we consider a particular situation in which retrodiction is a useful tool for finding atomic states at an earlier time. We assume that the initial atomic state is completely unknown, but the initial field is in a known coherent state. A measurement is made of the atomic state, and this result is used as a basis for retrodicting the atomic state at the preparation time. The retrodictive atomic density operator is given by equation (15). For ease of calculation we assume that the atomic state is measured to be in one of a pair of orthogonal states which span the two-level atomic basis, for example, the excited or ground state. We can calculate quantities based on other assumed bases using results for this one. The associated atomic POM element has unit trace and so the retrodictive atomic density operator immediately prior to measurement is simply the measurement POM element itself. Initially the field is in a coherent state, but the final state is unmeasured so the field measurement POM element is simply the unit operator for the field [12]. Thus equation (15) gives the initial retrodictive atomic density operator as

$$
\hat{\rho}_{\mathrm{a}}^{\mathrm{retr}}\left(t_{\mathrm{p}}\right) \propto\left\langle\alpha\left|\hat{U}^{\dagger}(\tau) \hat{\Pi}_{\mathrm{a}}\left(t_{\mathrm{m}}\right) \otimes \hat{1}_{\mathrm{f}} \hat{U}(\tau)\right| \alpha\right\rangle
$$

where $|\alpha\rangle$ is the coherent field state and $\hat{1}_{\mathrm{f}}$ is the unit state. It is relatively straightforward to compute the density matrix elements,

$$
\left\langle l\left|\hat{\rho}_{\mathrm{a}}^{\mathrm{retr}}\left(t_{\mathrm{p}}\right)\right| m\right\rangle \propto\langle\alpha|\left\langle l\left|\left\langle\hat{U}^{\dagger}(\tau) \hat{\Pi}_{\mathrm{a}}\left(t_{\mathrm{m}}\right) \otimes \hat{1}_{\mathrm{f}} \hat{U}(\tau) \mid m\right\rangle\right| \alpha\right\rangle
$$

where $l$ or $m$ can be either the ground or excited states, or one of any other pair of orthogonal states which span the two-level atomic basis. In fact $\hat{U}(\tau)|m\rangle|\alpha\rangle$ is simply the state that the 
atomic state $|m\rangle$ would have evolved into after a time $\tau$ in the predictive formalism. This allows us to use the well-known solution to the Jaynes-Cummings model [11],

$$
\hat{U}(\tau)|j\rangle|\alpha\rangle=\sum_{n=0}^{\infty}\left[c_{g, n}(\tau)|g\rangle|n\rangle+c_{e, n}(\tau)|e\rangle|n\rangle\right]
$$

where the states $|n\rangle$ are the photon number states and the ground and excited state amplitudes depend upon the coherent state expansion coefficients in the number state basis. The amplitudes are found to be

$$
\begin{aligned}
c_{g, n}(\tau) & =c_{g, n}(0)\left[\cos \frac{\Omega(n) \tau}{2}+\frac{i \Delta}{\Omega(n)} \sin \frac{\Omega(n) \tau}{2}\right] \\
& +c_{e, n-1}(0) \frac{2 \lambda n^{1 / 2}}{\Omega(n)} \sin \frac{\Omega(n) \tau}{2}, \\
c_{e, n-1}(\tau) & =c_{e, n-1}(0)\left[\cos \frac{\Omega(n) \tau}{2}-\frac{i \Delta}{\Omega(n)} \sin \frac{\Omega(n) \tau}{2}\right] \\
& -c_{g, n}(0) \frac{2 \lambda n^{1 / 2}}{\Omega(n)} \sin \frac{\Omega(n) \tau}{2},
\end{aligned}
$$

where $\Omega(n)=\left(\Delta^{2}+4 \lambda^{2} n\right)^{1 / 2}$ is the Rabi frequency, and $c_{g, n}(0)$ and $c_{e, n}(0)$ are the initial amplitudes, given by

$$
\begin{aligned}
& c_{g, n}(0)=a_{n} c_{g}(0) \\
& c_{e, n}(0)=a_{n} c_{e}(0) .
\end{aligned}
$$

These initial values are proportional to the number state expansion coefficients of the coherent state,

$$
a_{n}=\exp \left(-|\alpha|^{2} / 2\right) \frac{\alpha^{n}}{\sqrt{n}}
$$

We can use these formulae to calculate retrodictive matrix elements and probabilities, given that the atom was measured to be in a particular state. The calculations are relatively straightforward, and details are omitted.

We consider the case where the atom is known to have been prepared either in the excited state or the ground state with equal a priori probabilities. The appropriate preparation device operators in (4) are $|e\rangle\langle e| / 2$ and $|g\rangle\langle g| / 2$. Figure 1 shows a typical plot of the 
retrodictive conditional probability, in this case the probability that the atom was prepared in the ground state given that it was measured in the excited state. It shows retrodictive Rabi oscillations which collapse as the elapsed time before the measurement increases. If the preparation time was long enough before the measurement time the oscillations are seen to revive, just as in the predictive case. The characteristic revival time is the same as the predictive one. Thus "previvals" or "earlier revivals" in the preparation probability do exist.

Given that both the collapse time and the revival time are identical for the predictive and retrodictive evolutions one might think that the retrodictive evolution is simply the time-reverse of the predictive evolution. This is not the case, as is shown in figure 2, which depicts the evolution for a weaker coherent state. Here we compare predictive and retrodictive evolutions. Figure 2(a) shows the retrodictive conditional probability that the ground state was prepared given that the atom has been measured in the excited state. This can be compared with figures 2(b) and 2(c). These show, respectively, the predictive conditional probabilities that (b) the atom is measured in its ground state given that the excited state was prepared, and (c) the atom is measured in its excited state given that the ground state was prepared. The retrodictive evolution is clearly the time-reverse of neither of these two predictive evolutions .

This point can be illustrated more dramatically by considering other measured states. For example, for a high-amplitude coherent state, after (predictive) Rabi oscillations collapse, at a time $\tau=\pi /(2 \Omega(\bar{n}))$ the state of the system approximately factorises into uncoupled atomic and field states [9]. No matter what the initial prepared atomic state, after this period, which is half the revival time, the atom is prepared by the system in the state

$$
|-\rangle=\frac{1}{\sqrt{2}}\left(|g\rangle-e^{i \phi}|e\rangle\right),
$$

where $\phi$ is the phase of the coherent state amplitude $\alpha$ [13]. The predictive evolution of the state then consists of the revival of the Rabi oscillations.

On the other hand, if we measure the atom in this superposition state and try to retrodict the prepared state, the evolution is completely different. This is illustrated by figure 3, 
which is a plot of the excited state preparation probability. For a short delay there are Rabi oscillations which collapse, leaving a slow oscillation whose period is associated with the revival time of the system. If the delay is equal to half the revival time then the excited state probability passes through the value $1 / 2$. The atomic density operator becomes

$$
\hat{\rho}^{\operatorname{retr}}\left(\frac{\pi}{2 \Omega(\bar{n})}\right)=\frac{1}{2}[|e\rangle\langle e|+| g\rangle\langle g|],
$$

the no-information state. We say that the atomic state is unretrodictable at this time. The reason for this is simply that our measurement of the atom only provides information about the field at this time.

\section{CONCLUSIONS}

In this paper we have analysed the two-level atom interacting with a single-mode electromagnetic field in a coherent state from a retrodictive point of view. This system shows predictive collapses and revivals in the atomic state probabilities [8]. We have demonstrated the existence of retrodictive collapses and previvals of the Rabi oscillations in the atomic state probabilities. This follows on from our previous work which demonstrated the existence of retrodictive Rabi oscillations 《4].

We have shown that the retrodictive and predictive evolutions are different. The differences are most marked when either of two particular criteria are satisfied. Firstly, when the mean number of photons is low, for all measured atomic states, it becomes easy to differentiate between the predictive and retrodictive evolutions. Secondly, for high mean photon number, if the measured state is that to which all prepared states decay after a particular time then the retrodictive evolution takes on a strange character. There is a low-frequency retrodictive oscillation in the atomic state probability with a period equal to twice the revival time. Furthermore, when the time between preparation and measurement is equal to half the revival time the retrodictive state is unretrodictable. The probability that any one of a pair of states which span the atomic space was prepared will then be one half. 


\section{ACKNOWLEDGMENTS}

The authors would like to thank the United Kingdom Engineering and Physical Sciences Research Council and the Australian Research Council for financial support. SMB thanks the Royal Society of Edinburgh and the Scottish Office Executive Education and Lifelong Learning Department for the award of a Support Research Fellowship. 


\section{REFERENCES}

[1] Aharonov, Y., Bergmann, P.G. and Lebowitz, J.L., 1964, Phys. Rev. 134, B1410; Penfield, R.H., 1966, Am. J. Phys. 34, 422; Aharonov, Y. and Albert, D.Z., 1984, Phys. Rev. D 29, 223; Aharonov, Y. and Albert, D.Z., 1984, Phys. Rev. D 29, 228; Aharonov, Y. and Vaidman, L., 1991, J. Phys. A:Math. Gen. 24, 2315; Pegg, D.T. and Barnett, S.M., 1999, Quantum Semiclass. Optics 1, 442.

[2] Box, G.E.P. and Tiao, G.C. 1973, Bayesian Inference in Statistical Analysis (Sydney: Addison-Wesley).

[3] Barnett, S.M., Pegg D.T. and Jeffers, J., 2000, J. Mod. Opt. 47, 1779.

[4] Barnett, S.M., Pegg, D.T., Jeffers, J. and Jedrkiewicz, O., 2000, J. Phys. B: At. Mol. Opt. Phys. 33, 3047.

[5] Barnett, S.M., Pegg, D.T., Jeffers, J., Jedrkiewicz, O. and Loudon, R., 2000, Phys. Rev. A 62, 022313.

[6] Barnett, S.M., Pegg, D.T., Jeffers, J. and Jedrkiewicz, O., 2000, Phys. Rev. Lett. 86, 2455 .

[7] Helstrom, C.W., 1976, Quantum Detection and Estimation Theory (New York: Academic Press).

[8] Eberly, J.H., Narozny N.B., and Sanchez-Mondragon, J.J., 1980, Phys. Rev. Lett. 44, 1323; Narozny, N.B., Sanchez-Mondragon, J.J. and Eberly, J.H., 1981, Phys. Rev. A 23, 236.

[9] Gea-Banacloche, J., 1990, Phys. Rev. Lett. 65, 3385.

[10] Shore, B.W. and Knight, P.L., 1993, J. Mod. Opt. 40, 1195.

[11] See for example, Barnett, S.M. and Radmore, P.M., 1997, Methods in Theoretical Quantum Optics, (Oxford: Oxford University Press). 
[12] This is because the act of not measuring the field is equivalent to performing a measurement with only one possible outcome. The probability for this outcome is, of course, unity for all possible states and so the POM element is the identity

[13] This state is not the one found in reference [9], a difference which can be explained by the fact that our Hamiltonian has a slightly different form. 


\section{FIGURE CAPTIONS}

Figure 1: A plot of the retrodictive conditional probability that the atom was prepared in the ground state given a later measurement in the excited state as a function of the normalised difference between preparation and measurement times $\lambda \tau$. Parameters: detuning $\Delta=0$ and coherent state amplitude $\alpha=5$.

Figure 2: (a) Same plot as figure 1, but with coherent state amplitude $\alpha=1.4$. (b) A plot of the predictive conditional probability that the atom was measured in the ground state given that it was prepared in the excited state as a function of $\lambda \tau$. Parameters are as for (a). (c) A plot of the predictive conditional probability that the atom was measured in the excited state given that it was prepared in the ground state as a function of $\lambda \tau$. Parameters are as for (a).

Figure 3: A plot of the retrodictive conditional probability that the atom was prepared

in the excited state given a later measurement in the superposition state $\frac{1}{\sqrt{2}}\left(|g\rangle-e^{i \phi}|e\rangle\right)$ as a function of $\tau$. The parameters are as in figure 1 . 


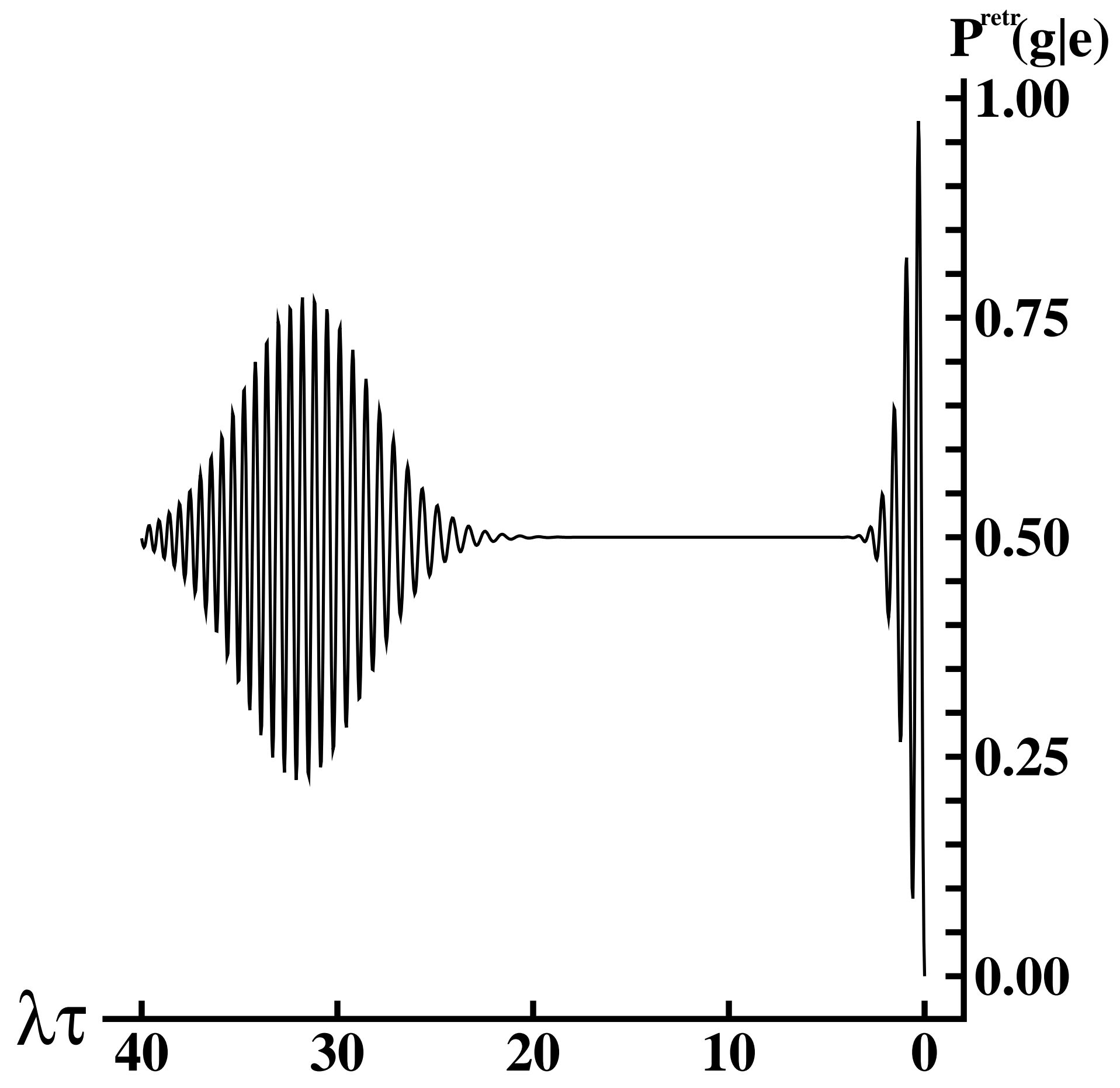




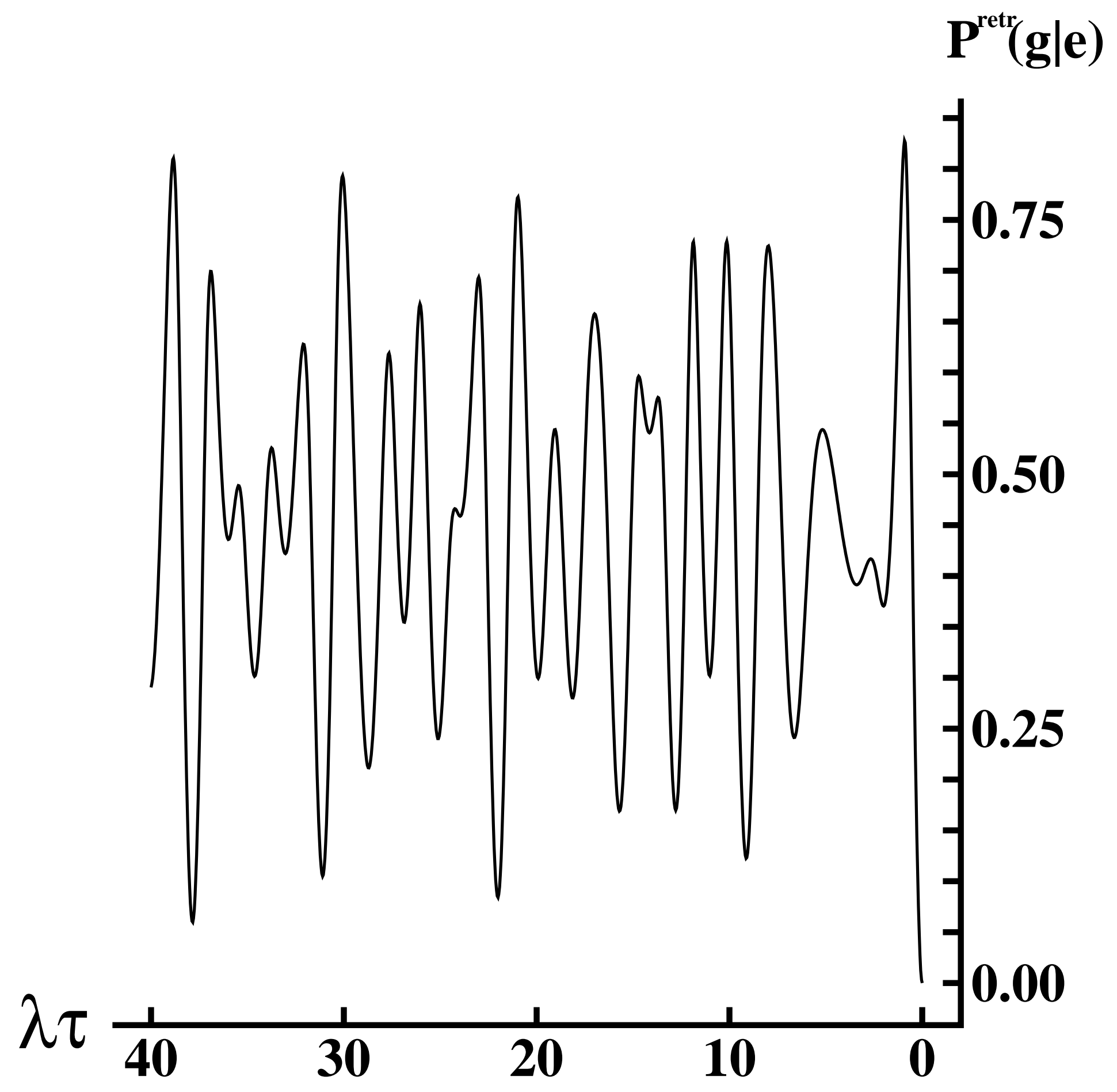




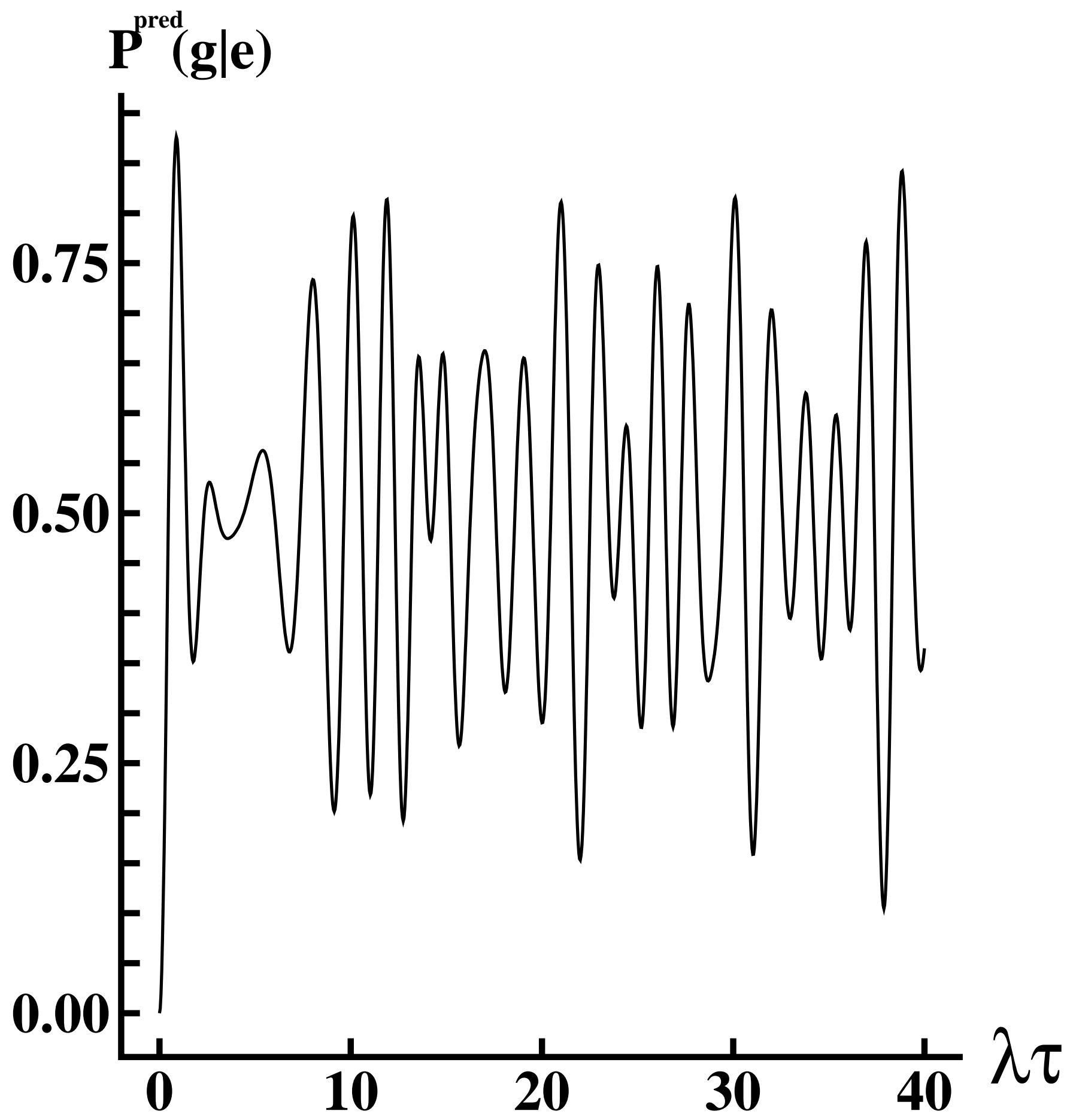




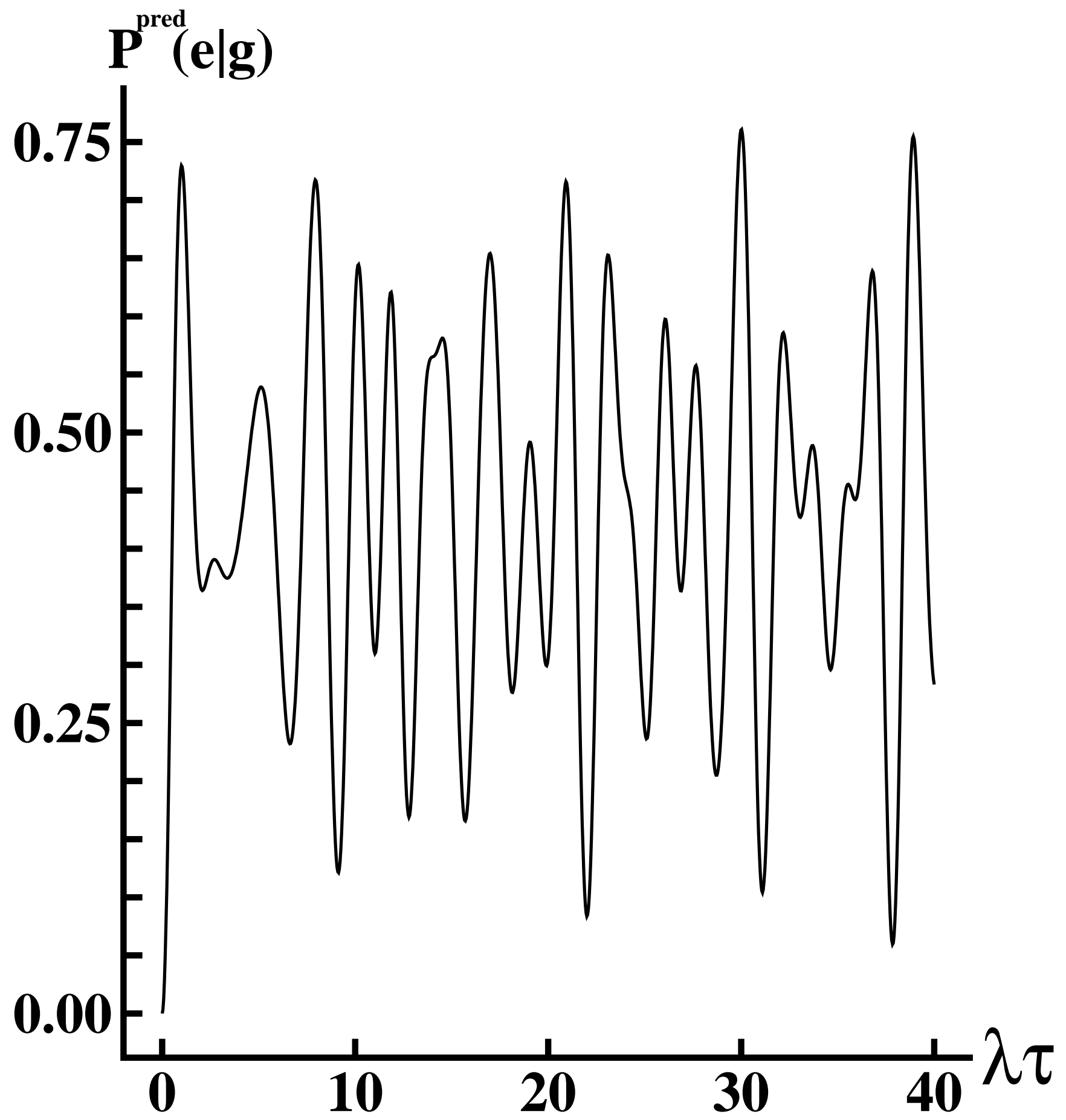




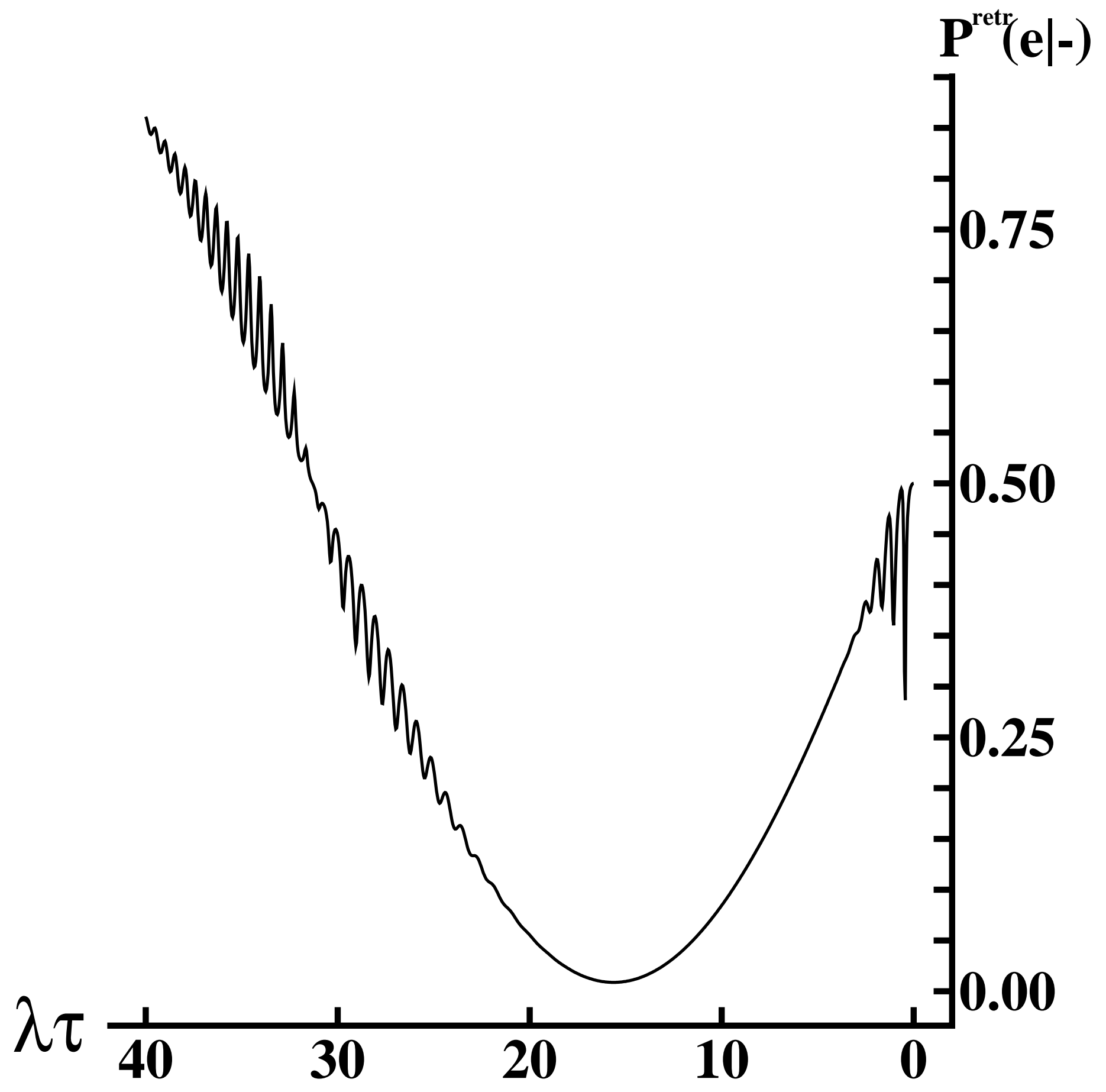

